\title{
An integrative bioinformatic predictor of protein sub-cellular localisation in malaria
}

\author{
Ben J Woodcroft ${ }^{1,2}$, Robert Radloff ${ }^{1,3}$, Lee M Yeoh ${ }^{1,4}$, Kristie-Lee Scanlon ${ }^{1}$, Maria A Doyle ${ }^{1,5}$, Giel G van Dooren ${ }^{4}$, \\ Geoffrey I McFadden ${ }^{4}$, Christopher J Tonkin², Terence P Speed ${ }^{2}$, Stuart A Ralph ${ }^{{ }^{*}}$ \\ From Seventh International Society for Computational Biology (ISCB) Student Council Symposium 2011 \\ Vienna, Austria. 15 July 2011
}

\section{Background}

The malarial parasite Plasmodium falciparum remains a leading international cause of mortality, with almost a million deaths each year. Determination of protein subcellular localisation remains a challenge in Plasmodium parasites due to their evolutionary distance from wellstudied model organisms, and limited efficiency of appropriate molecular tools. However, abundant large scale systems biology information exist for several Plasmodium species as well as other apicomplexan parasites, including full genomic DNA sequences, plus data sets relating to the transcriptome, protein expression and interactions, polymorphisms and phyletic profiles. To date, most bioinformatic predictors of sub-cellular localisation use sequence information exclusively without consideration for other data sets.

\section{Materials and methods}

We developed the first global bioinformatic predictor of sub-cellular localisation in Plasmodium falciparum (called Plasmarithm) that predicts localisation for multiple cellular compartments using a variety of post-genomic information types.

\section{Results and conclusions}

We identified several non-sequence data types that are predictive of localisation, including phyletic distribution and transcript abundance at specific life stages. We performed a comprehensive literature survey of the phylum Apicomplexa to construct a database of $>850$ recorded protein localisations curated from $>700$ separate publications. The database, called ApiLoc (freely available at

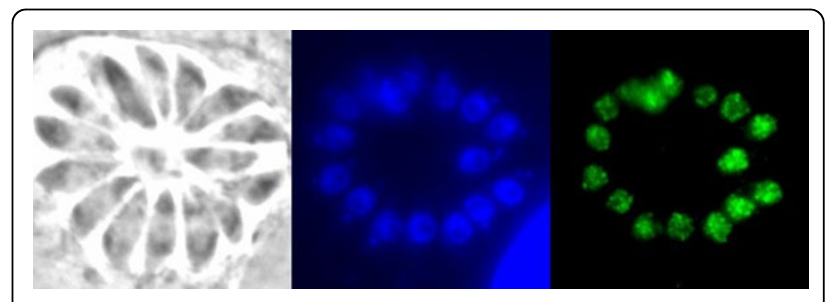

Figure 1 Experimental confirmation of a Plasmarithm localisation prediction. A P. falciparum protein (PlasmoDB ID PFE0425w) was predicted to be nuclear, and here the HA tagged T. gondii orthologue (ToxoDB ID TGME49_044840) is shown exhibiting a nuclear localisation. The panels show bright field, Hoechst (nuclear) stained, and HA tagged TGME49_044840 images, respectively.

http://apiloc.bio21.unimelb.edu.au), was used to improve the accuracy of our predictor. We achieved an overall accuracy of $\sim 60 \%$ on a seven class problem, where a number of the classes have not previously been predicted. To further validate these in-silico analyses, we have experimentally verified localisations of a number of hypothetical proteins in the related apicomplexan Toxoplasma gondii (Figure 1).

\section{Author details}

'Department of Biochemistry \& Molecular Biology, Bio21 Molecular Science and Biotechnology Institute, University of Melbourne, Australia. ${ }^{2}$ The Walter and Eliza Hall Institute of Medical Research, Melbourne, Australia. ${ }^{3}$ Institute for Biochemistry, University of Stuttgart, Stuttgart, Germany. ${ }^{4}$ Plant Cell Biology Research Centre, School of Botany, University of Melbourne, Melbourne, Australia. ${ }^{5}$ Bioinformatics Core Facility, Peter MacCallum Cancer Centre, Melbourne, Australia.

Published: 21 November 2011

\footnotetext{
* Correspondence: saralph@unimelb.edu.au

${ }^{1}$ Department of Biochemistry \& Molecular Biology, Bio21 Molecular Science and Biotechnology Institute, University of Melbourne, Australia Full list of author information is available at the end of the article
}

doi:10.1186/1471-2105-12-S11-A6

Cite this article as: Woodcroft et al:: An integrative bioinformatic predictor of protein sub-cellular localisation in malaria. $B M C$ Bioinformatics 2011 12(Suppl 11):A6. 